Canad. Math. Bull. Vol. 19 (4), 1976

\title{
BEATTY SEQUENCES, CONTINUED FRACTIONS, AND CERTAIN SHIFT OPERATORS
}

\author{
BY
}

\author{
KENNETH B. STOLARSKY
}

\begin{abstract}
Let $\theta=\theta(k)$ be the positive root of $\theta^{2}+(k-2) \theta-$ $k=0$. Let $f(n)=[(n+1) \theta]-[n \theta]$ for positive integers $n$, where $[x]$ denotes the greatest integer in $x$. Then the elements of the infinite sequence $(f(1), f(2), f(3), \ldots)$ can be rapidly generated from the finite sequence $(f(1), f(2), \ldots, f(k))$ by means of certain shift operators. For $k=1$ we can generate (the characteristic function of) the sequence $[n \theta]$ itself in this manner.
\end{abstract}

1. Introduction. Since the "golden mean" $\alpha=\frac{1}{2}(1+\sqrt{ } 5)$ has the expansion $1+\frac{1}{1+} \frac{1}{1+} \cdots$ as a simple continued fraction, it is readily associated with the Fibonacci sequence $1,1,2,3,5,8, \ldots$ We shall show there is an apparently very different way in which $\alpha$ is associated with this sequence. Let $101010 \ldots$ be the sequence consisting of the 2 numbers 0,1 repeated infinitely often (in that order). If we repeat the first 3 numbers of this sequence infinitely often we get $101101101 \cdots$. If we similarly repeat the first 5 numbers here, we get $1011010110 \cdots$; for $\mathbf{8}$ we get $1011010110110101 \cdots$. It is clear that this process "converges" (see $\$ 2$ for a precise definition) to a sequence $a_{1} a_{2} a_{3} \ldots$ where $a_{i}$ is 0 or 1 .

THeOREM 1. Let $[x]$ denote the largest integer not exceeding $x$. Then $a_{n}=1$ if $n=[m \alpha]$ for some integer $m$, and $a_{n}=0$ otherwise.

We shall find a similar phenomenon (Theorem 2 of §3) for an infinite class of quadratic irrationalities, and show that it is in fact a consequence of well known properties of simple continued fractions. Theorem 1 is deduced from the case $k=1$ of Theorem 2 in $\S 4$. In $\S 5$ we shall show that Theorem 2 can also be deduced from a general result of Markoff on simple continued fractions. The author believes that the list of references at the end of this paper is the first reasonably complete bibliography on Beatty sequences and related topics.

Although our subject has roots in [6] and [48], it was the striking problem posed by the late Professor Samuel Beatty of Toronto in [5] that brought it to

Received by the editors March 17, 1975 and, in revised form, August 13, 1975.

AMS (MOS) subject classifications (1970). Primary 10A35, 10A30, 10F20.

Key words and phrases. Beatty sequences, complementary sequences of natural numbers, continued fractions, Fibonacci sequence, golden mean, greatest integer function, shift operators, Wythoff's game. 
the attention of the mathematical community at large. The topic was then disseminated and pursued on a worldwide basis. As items [2, 3, 5, 18-21, 25, $34,44,46]$ indicate only inadequately, this was done nowhere more vigorously than in Beatty's native Canada.

Let $\theta=1+1 / k+1 / k+\cdots=1+\frac{1}{2}\left\{\sqrt{ }\left(k^{2}+4\right)-k\right\}$. The Beatty sequence $a_{n}=$ [ $n \theta$ ], where $1 \leq n<\infty$, is central to the theory of Wythoff's game and its generalizations $[1,3,18,19,21,22,29,40,41,43,67,68]$. Theorem 2 of $\S 3$ asserts that the first difference of this sequence can be generated very rapidly from the $k+1$ tuple $([\theta],[2 \theta], \ldots,[(k+1) \theta])$ by means of a certain sequence of shift operations. In fact, let $f(n)=[(n+1) \theta]-[n \theta]$ and let

$$
F=(f(1), f(2), f(3), \ldots) \text {. }
$$

Then $F$ is a fixed point of a certain transformation $T$ such that (i) $T(F)$ depends only on the first $k$ elements of $F$, and (ii) $T$ is the limit of a sequence of shift operators, defined in $\$ 2$, corresponding to elements of the sequence defined by $t_{0}=1, t_{1}=k$, and $t_{n}=k t_{n-1}+t_{n-2}$ for $n \geq 2$.

Our proof hinges on the following well known result.

Lemma. Let $n \geq 1$. Let $p_{n} / q_{n}$ be the $n$-th convergent to the irrational number $\phi$. If $a$ and $b$ are integers with $b>0$ and $|\phi b-a|<\left|\phi q_{n}-p_{n}\right|$, then $b \geq q_{n+1}$.

A proof can be found, for example, in [53, p. 163].

The standard way of generating $a_{n}$ is as follows. Let $a_{1}=1$ and $b_{1}=k+1$. After $a_{i}$ and $b_{i}$ have been generated for $i<n$, let $a_{n}$ be the smallest positive integer which is distinct from all these $a_{i}$ and $b_{i}$, with $i<n$, and let $b_{n}=a_{n}+n k$. Since $\theta^{-1}+(\theta-k)^{-1}=1$ we have [see, e.g., 5, 21, or 52 pp. 34-35] that $a_{n}=[n \theta]$ and $b_{n}=[n(\theta+k)]$. Thus $\left\{a_{n}\right\}$ and $\left\{b_{n}\right\}$ are complementary Beatty sequences.

2. Certain shift operators. Let $C=\left(c_{1}, c_{2}, c_{3}, \ldots\right)$ be a finite or infinite sequence of integers. Let $e_{i}(C)=c_{i}$ be the $i$ th element of the sequence $C$. If $C_{j}=\left(c_{1 j}, c_{2 j}, \ldots\right)$ we say that $\lim _{j \rightarrow \infty} C_{j}=C$ if $e_{i}\left(C_{j}\right)=c_{i}$ for $j$ sufficiently large.

If $C=\left(c_{1}, \ldots, c_{n}\right)$ and $D=\left(d_{1}, \ldots, d_{m}\right)$ are finite sequences, we denote by $C D$ their juxtaposition; i.e.,

$$
C D=\left(c_{1}, \ldots, c_{n}, d_{1}, \ldots, d_{m}\right) .
$$

We allow infinite juxtaposition; thus $D^{\infty}$ is the infinite sequence of period $m$ whose first $m$ members are $d_{1}, \ldots, d_{m}$. We denote by $(C, t)$ the finite sequence whose first $t$ elements are $c_{1}, \ldots, c_{t}$; i.e.,

$$
(C, t)=\left(c_{1}, \ldots, c_{t}\right) \text {. }
$$

Now let $T=\left\{t_{n}\right\}_{n=1}^{\infty}$ be a sequence of strictly increasing positive integers. Define $T_{n}(C)$ inductively by

$$
T_{1}(C)=\left(C, t_{1}\right)^{\infty}
$$


and

$$
T_{n}(C)=\left(T_{n-1}(C), t_{n}\right)^{\infty}
$$

Set

$$
T(C)=\lim _{n \rightarrow \infty} T_{n}(C)
$$

the limit obviously exists. Note that if the sequences $C$ and $C^{\prime}$ have the same initial $t_{1}$ members, then $T_{1}(C)=T_{1}\left(C^{\prime}\right)$ and hence $T(C)=T\left(C^{\prime}\right)$.

For example, let $C=(1,0)^{\infty}$ and let $T=(2,3,5,8,13, \ldots)$ be the sequence of Fibonacci numbers that exceed 1 . Then $T_{1}(C)=(1,0)^{\infty}, T_{2}(C)=(1,0,1)^{\infty}$, $T_{3}(C)=(1,0,1,1,0)^{\infty}$, and $T_{4}(C)=(1,0,1,1,0,1,0,1)^{\infty}$.

3. The main result. Recall that $\theta=1+1 / k+1 / k+\cdots$ and that $F=$ $(f(1), f(2), f(3), \ldots)$ where $f(n)=[(n+1) \theta]-[n \theta]$.

Theorem 2. Let $k \geq 2$ be a positive integer and let $T=\left\{t_{n}\right\}_{n=1}^{\infty}$ where $t_{0}=1$, $t_{1}=k$, and $t_{n}=k t_{n-1}+t_{n-2}$ for $n \geq 2$. Then

$$
T(F)=F .
$$

If $k=1$ the result is valid for $T=\left\{t_{n}\right\}_{n=2}^{\infty}$.

Before giving a proof, we require some notation and several lemmas. Given an irrational continued fraction $x=a_{0}+1 / a_{1}+1 / a_{2}+\cdots$ define (as usual) sequences $\left\{p_{n}\right\}$ and $\left\{q_{n}\right\}$ inductively as follows:

$$
\begin{array}{lllll}
p_{-2}=0 & p_{-1}=1 & p_{n}=a_{n} p_{n-1}+p_{n-2} & \text { for } & n \geq 0, \\
q_{-2}=1 & q_{-1}=0 & q_{n}=\dot{a}_{n} q_{n-1}+q_{n-2} & \text { for } & n \geq 0 .
\end{array}
$$

Let $r_{j}=p_{j} / q_{j}$.

LEMMA 3.1. For $\varphi=\theta+k-1$ we have $a_{i}=k$ for all $k$, so $p_{j}=t_{j+1}$ and $q_{j}=t_{j}$.

LEMMA 3.2. We have $t_{2 j} \varphi-t_{2 j+1}>0$ and $t_{2 j+2}-\varphi t_{2 j+1}>0$ for $j \geq 0$.

Proof. For $j \geq 0$, it is well known that $r_{2 j}<x<r_{2 j+1}[53, p$. 156], so this follows from Lemma 3.1.

LEMMA 3.3

(i) If $t_{2 j+2}>m$, then

$$
1>m \varphi-[m \varphi] \geq t_{2 j+2}-\varphi t_{2 j+1}>0 . \quad(j \geq 0)
$$

(ii) If $t_{2 j+1}>m$, then

$$
1+[m \varphi]-m \varphi \geq t_{2 j} \varphi-t_{2 j+1}>0 . \quad(j \geq 1) .
$$

Proof. This is an immediate consequence of Lemmas 3.1, 3.2, and the contrapositive of the Lemma quoted in the introduction; simply let $b=m$ and take $a=[m \varphi]$ for (i) and $a=1+[m \varphi]$ for (ii). 
LEMMA 3.4

(i) If $t_{2 j+2}>m$, then

$$
t_{2 j+2}+[m \varphi]=\left[\left(t_{2 j+1}+m\right) \varphi\right] . \quad(j \geq 0)
$$

(ii) If $t_{2 j+1}>m$, then

$$
t_{2 j+1}+[m \varphi]=\left[\left(t_{2 j}+m\right) \varphi\right] . \quad(j \geq 1) .
$$

Proof. Let

and

$$
r=t_{2 j+1} \varphi-t_{2 j+2}+m \varphi-[m \varphi]
$$

$$
s=m \varphi-[m \varphi]+t_{2 j} \varphi-t_{2 j+1} .
$$

If $t_{2 j+2}>m$, Lemma 3.3(i) shows that $[r]=0$. But upon adding [r] to the left side of (i), we get the right side. If $t_{2 j+1}>m$, Lemma 3.3(ii) shows that $[s]=0$. But upon adding $[s]$ to the left side of (ii), we get the right side. Thus the lemma is established.

Thus we have proved

Corollary 3.1. If $j \geq 1$ and $m<t_{j+1}$, then

$$
\left[\left(t_{j}+m\right) \varphi\right]=t_{j+1}+[m \varphi] \text {. }
$$

We now establish

Corollary 3.2. If $j \geq 1$ and $n+1<t_{j+1}$, then

$$
f\left(n+t_{j}\right)=f(n) \text {. }
$$

Proof. Recall that $\varphi=\theta+k-1$. Thus

$$
\begin{aligned}
f\left(n+t_{j}\right)=\left[\left(n+1+t_{j}\right) \theta\right]-\left[\left(n+t_{j}\right) \theta\right] \\
=\left[\left(n+1+t_{j}\right) \varphi\right]-(k-1)\left(n+1+t_{j}\right)-\left[\left(n+t_{j}\right) \varphi\right]+\left(n+t_{j}\right)(k-1) .
\end{aligned}
$$

By Corollary 3.1 the right side of the above is

$$
\begin{aligned}
t_{j+1}+[(n+1) \varphi]-\left(t_{j+1}+[n \varphi]\right) & -(k-1) \\
= & {[(n+1) \varphi]-[n \varphi]-(k-1)=[(n+1) \theta]-[n \theta]=f(n) . }
\end{aligned}
$$

This proves Corollary 3.2. We comment that it is not always valid for $n+1=t_{j+1}$.

We can now prove the theorem. Say $k \geq 2$, and note that $n+1<t_{j+1}$ means $n \leq t_{j+1}-2$. The sequence $F$ certainly agrees with itself in the first $k$ places. Since $t_{1}=k$, the sequence $T_{1}(F)$, by Corollary 3.2, will agree with $F$ in the first $t_{1}+t_{2}-2$ places. In general, say $T_{j}(F)$ agrees with $F$ in the first $t_{j+1}+t_{j}-2$ places. Since $t_{j+1} \leq t_{j+1}+t_{j}-2$, these places completely determine $T_{j+1}(F)$. By Corollary 3.2 , the sequence $T_{j+1}(F)$ will agree with $F$ in the first $t_{j+2}+t_{j+1}-2$ places. Thus $T(F)=F$. The proof for $k=1$ is the same, aside from the initialization. 
4. The "golden mean" Beatty sequence. We shall now prove Theorem 1. For $h \geq 1$ let $a_{h}=[h \alpha]$ and $b_{h}=a_{h}+h$ where $\alpha=\frac{1}{2}(1+\sqrt{5})$. Thus $\left\{a_{h}\right\}=$ $(1,3,4,6,8,9, \ldots)$.

LEMMA 4.1. The inequality $a_{h} \leq n$ holds if and only if $b_{h} \leq a_{n+1}$.

Proof. If $a_{h} \leq n$ then $h \alpha<n+1$, so

$$
b_{h}=[h(\alpha+1)]=\left[h \alpha^{2}\right] \leq[(n+1) \alpha]=a_{n+1} .
$$

On the other hand, if $b_{h} \leq a_{n+1}$ then

$$
b_{h}=[h(\alpha+1)]<(n+1) \alpha .
$$

We now claim that $h \alpha<n+1$. If not, then $h \alpha+h \geq n+1+h$ and $b_{h}=$ $[h \alpha+h] \geq n+1+h$. From this and (4.2), we find

$$
b_{h} \alpha>b_{h}+h \alpha \geq b_{h}+n+1
$$

and

$$
b_{h} \alpha^{2}>b_{h} \alpha+b_{h},
$$

a contradiction to the fact that $\alpha^{2}=\alpha+1$. Hence $h \alpha<n+1$, so $a_{h} \leq n$ and the proof is complete.

Now let $A(n)$ be the number of $a_{h}$ such that $a_{h} \leq n$.

Lemma 4.2. The sequence $a_{k}$ can be defined inductively by $a_{1}=1$ and $a_{n+1}=n+1+A(n)$.

Proof. It is clear that $1 \leq a_{n+1}-a_{n} \leq 2$ and that

$$
a_{n+1}=1+\sum_{h=1}^{n}\left(a_{h+1}-a_{h}\right) .
$$

Each term of the above sum is either 1 or 2 , so $a_{n+1}=1+n+K(n)$ where $K(n)$ is the number of twos. It is clear from the standard way of generating the $a_{k}$ (see $\S 1$ ) that $K(n)$ is the number of $b_{i}$ such that $b_{i} \leq a_{n+1}$. But by Lemma 4.1, we have that $K(n)=A(n)$. This completes the proof.

We can now prove Theorem 1. By Lemma 4.2 we have $a_{n+1}-a_{n}=$ $1+[A(n)-A(n-1)]$. The expression in brackets is 1 if $n=[h \alpha]$ for some integer $h$ and 0 otherwise. Thus the sequence of differences is, aside from an additive constant of 1 (which doesn't really matter), the characteristic function of the sequence $[h \alpha]$. But we can apply the Theorem of $\S 3$ to the sequence of differences. This completes the proof.

5. A proof by Markoff's theorem. Let $\xi$ be a real number such that $0<\xi<1$, and set $f(m)=[(m+1) \xi]-[m \xi]$. Let

$$
\xi=\frac{1}{k+} \frac{1}{k_{1}+} \frac{1}{k_{2}+} \ldots
$$


be the expansion of $\xi$ into a simple contained fraction. Let $S(c, d)$ be the free semigroup on the letters $c$ and $d$.

Definition 5.1. For $m \geq 1$, let $c_{m}$ and $d_{m}$ be the sequences of elements from $S(c, d)$ defined inductively by

$$
c_{1}=c^{k-1} d, \quad d_{1}=c^{k} d
$$

and

$$
c_{m+1}=c_{m}^{k_{m}-1} d_{m}, \quad d_{m+1}=c_{m}^{k_{m}} d_{m}
$$

for $m \geq 1$. Let

$$
x=c_{1} c_{2} c_{3} \cdots=\prod_{i=1}^{\infty} c_{i}
$$

Now write

$$
x=\prod_{i=1}^{\infty} g_{i}
$$

where each $g_{i}$ is either $c$ or $d$.

Theorem (MARkoff). For $m \geq 1$ we have $f(m)=0$ if $g_{m}=c$ and $f(m)=1$ if $g_{m}=d$.

This result was originally obtained by A. A. Markoff [48] in his investigation of a problem of the astronomer J. Bernoulli [6]. The above formulation is from Venkov [65, pp. 65-68], who also gives a proof. We shall apply this to the case

$$
\xi=\theta-1=\frac{1}{k+} \frac{1}{k+} \frac{1}{k+} \cdots
$$

to obtain an alternate proof of our own result.

Definition 5.2. For $w \in S(c, d)$, let $\bar{w}$ be the word obtained from $w$ by replacing each $c$ by $c^{k-1} d$ and each $d$ by $c^{k-1} d c$. For example, $\overline{c d}=$ $c^{k-1} d c^{k-1} d c$.

It is clear that $\overline{w_{1} w_{2}}=\overline{w_{1}} \cdot \overline{w_{2}}$ for words $w_{1}$ and $w_{2}$ in $S(c, d)$. It is also clear that $\bar{w}$ makes sense for infinite words $w=a_{1} a_{2} a_{3} \cdots$ where each $a_{i}$ is either $c$ or $d$.

REMARK. We have from (5.2), (5.3), and (5.6) that

$$
c_{1}=c^{k-1} d, \quad c_{2}=\left(c^{k-1} d\right)^{k-1} c^{k} d
$$

and

$$
c_{n+2}=c_{n+1}^{k-1} c_{n} c_{n+1} \text { for } n \geq 1 .
$$

For the next lemma, consider $S(c, d)$ as imbedded in $G(c, d)$, the free group on $c$ and $d$. 
Lemma 5.1. For $r \geq 1$ we have

$$
c_{1} c_{r+1} c_{1}^{-1}=\overline{c_{r}} .
$$

Proof. This is true for $r=1$, since

$$
\begin{aligned}
c_{1} c_{2} c_{1}^{-1}=\left(c^{k-1} d\right)^{k} & c^{k} d\left(c^{k-1} d\right)^{-1}=\left(c^{k-1} d\right)^{k} c \\
= & \left(c^{k-1} d\right)^{k-1} c^{k-1} d c=\bar{c}^{k-1} \bar{d}=\overline{c_{1}} .
\end{aligned}
$$

Say it is true for $r \leq n$. Then

$$
\begin{gathered}
c_{1} c_{n+2} c_{1}^{-1}=\left(c_{1} c_{n+1} c_{1}^{-1}\right)^{k-1}\left(c_{1} c_{n} c_{1}^{-1}\right)\left(c_{1} c_{n+1} c_{1}^{-1}\right) \\
=\left(\overline{c_{n}^{k-1} c_{n-1} c_{n}}\right)=\bar{c}_{n+1}
\end{gathered}
$$

and the lemma is proved.

LEMMA 5.2. For $r \geq 1$, we have

$$
\overline{\left(c_{1} \cdots c_{r}\right)} c_{1}=c_{1} c_{2} \cdots c_{r+1} \text {. }
$$

Proof. Multiply both sides by $c_{1}^{-1}$ on the right, and apply the previous lemma.

Definition 5.3. Let $w_{1}=c_{1}$ and $w_{n+1}=\overline{w_{n}}$. Let $\ell\left(w_{n}\right)$ denote the length of $w_{n}$; that is, the number of letters in $w_{n}$. For example, $\ell\left(c^{3} d\right)=4$.

LEMMA 5.3. There is a unique infinite word $w$ such that (i) $w$ has $c_{1}$ as an initial segment, and (ii) $\bar{w}=w$. In fact, $w=x$.

Proof. It is clear that the lengths of the words $w_{n}$ are strictly increasing. Since each $w_{n}$ must be an initial segment of $w$, the infinite word $w$ is unique (if it exists). On the other hand, the infinite word $x$ defined in (5.4) begins with $c_{1}$, and $\bar{x}=x$ follows from Lemma 5.2. Take $w=x$. This completes the proof.

We thus have that the sequence $\left\{w_{n}\right\}$ converges to $w$ (with essentially the same notion of convergence as in $\S 2$ ).

LEMMA 5.4. For $n \geq 2$, we have $w_{n+1}=w_{n}^{k} w_{n-1}$.

Proof. Define $w_{0}=c$. Then $w_{m+1}=\bar{w}_{m}$ for $m \geq 0$. Since $w_{2}=\left(c^{k-1} d\right)^{k} c=$ $w_{1}^{k} w_{0}$, the lemma is valid for $n=1$. Say it is true for $n \leq m$. Then

$$
w_{m+2}=\bar{w}_{m+1}=\overline{w_{m}^{k} w_{m-1}}=w_{m+1}^{k} w_{m}
$$

and the lemma is proved.

Since $w_{n-1}=w_{n}\left(w_{n-1}^{k-1} w_{n-2}\right)^{-1}$, we have

$$
w_{n+1}=w_{n}^{k+1}\left(w_{n-1}^{k-1} w_{n-2}\right)^{-1} \text {. }
$$

This shows that $w_{n+1}$ is an initial segment of the infinite word formed by repeated juxtaposition of $w_{n}$. Thus we once again have our result on shift operators. 
6. Bibliography. Let $\alpha>0$ and $\beta$ be real numbers, and let $[x]$ denote the greatest integer in $x$. This bibliography attempts to list all papers concerning the sequence $[\alpha n+\beta], n=1,2,3, \ldots$, which can be considered as "descendants" of Beatty [5], Markoff [48], and Wythoff [67]. I believe it is complete at least up to 1972 , but perhaps this is wishful thinking. I would greatly appreciate any further references. A fairly complete bibliography of this area should be useful, since it is notorious for the frequency with which known facts are rediscovered and republished.

A quick overview of the subject is given in Coxeter [21]. For more detailed introductions to Beatty sequences and Wythoff's game, see Niven [52, pp. 34-45] and Connell [18] respectively. Most (if not all) of the known facts about Bernoulli sequences can be found in Venkov [65, pp. 65-68] and Uspensky $[16,17]$. For research areas which are presently active, see Fraenkel [25-30] and Cohn [14-16].

The following question seems untouched: for an irrational $\alpha>0$, what can be said about the second difference of $\left[\alpha n^{2}+\beta\right]$ ?

The author thanks A. S. Fraenkel for providing him with references $[1,6$, 27-31, 36, 38, 48, 65] and H. Cohn for [14-16].

\section{REFERENCES}

1. W. Ahrens, Mathematische Unterhaltungen und Spiele, vol. 1, 2nd ed., Teubner, Leipzig, 1910 (pp. 84-88).

2. M. Angel, Partitions of the natural numbers, Canad. Math. Bull. 7 (1964), 219-236. MR 28 \#5030.

3. W. W. R. Ball, Mathematical Recreations and Essays, revised by H. S. M. Coxeter, MacMillan, New York, 1939 (pp. 36-40).

4. Th. Bang, On the sequence $[n \alpha], n=1,2, \ldots$, Math. Scand. 5 (1957), 69-76. MR 19 (1958), p. 1159.

5. S. Beatty, Problem 3173, Amer. Math. Monthly 33 (1926), 159. Solutions, ibid., 34 (1927), 159.

6. J. Bernoulli III, Sur une nouvelle espèce de calcul, Recueil pour les astronomes, vols. 1, 2, Berlin, 1772.

7. R. Bricard, Sur un problème relatif aux nombres incommensurables, Nouv. Ann. de Math. (6) 1 (1926), 100-103.

8. L. Carlitz, V. E. Hoggatt, Jr., and R. Scoville, Fibonacci representations, Fib. Quart. 10 (1972), 1-28.

9. - - - and - Lucas representations, Fib. Quart. 10 (1972), 29-42, 70, 112.

10. - - - and - Fibonacci representations of higher order, Fib. Quart. 10 (1972), 71-80.

11. - - - , and - Fibonacci representations of higher order II, Fib. Quart. 10 (1972), 71-80.

12. ——, - - and - Pellian representations, Fib. Quart. 10 (1972), 449-488; Addendum, ibid., 527-530. MR 47 \#8420.

13. L. Carlitz, R. Scoville, and T. Vaughan, Some arithmetic functions related to Fibonacci numbers, Fib. Quart. 11 (1973), 337-386. MR 48 \#10969.

14. H. Cohn, Representation of Markoff's binary quadratic forms by geodesics on a perforated torus, Acta Arith. 18 (1971), 125-136. MR 44 \#5277.

15. —, Markoff forms and primitive words, Math. Ann. 196 (1972), 8-22. MR 45 \#6899.

16. - Some direct limits of primitive homotopy words and of Markoff geodesics, esp. pp. 97-98, 
in Discontinuous Groups and Riemann Surfaces, L. Greenberg ed., Ann. of Math. Studies 79,

Princeton University Press, Princeton, 1974.

17. — Problem E2544, Amer. Math. Monthly 82 (1975), 660. Solution, ibid., 83 (1976), 745-747.

18. I. G. Connell, A generalization of Wythoff's game, Canad. Math. Bull. 2 (1959), 181-190.

19. — Some properties of Beatty sequences I, Canad. Math. Bull. 2 (1959), 190-197. MR \#7805.

20. - Some properties of Beatty sequences II, Canad. Math. Bull. 3 (1960), 17-22. MR 22 \#1558.

21. H. S. M. Coxeter, The golden section, phyllotaxis and Wythoff's game, Scripta Math. 19 (1953), 135-143. MR 15 (1954), p. 246.

22. A. P. Domoryad, Mathematical Games and Diversions (Russian), Gosudarstv. Izdat. FizMat. Lit., Moscow, 1961 (pp. 59-67). MR 23 \#A761.

23. K. Fan, Problem 4399, Amer. Math. Monthly 57 (1950), 343. Solutions, ibid., 59 (1952), 48-49.

24. Faucheux, Sur une question concernant des suites de nombres incommensurables, Nouv. Ann. de Math. (6) 1 (1926), 237-239.

25. A. S. Feaenkel, The bracket function and complementary sets of integers, Can. J. Math. 21 (1969), 6-27. MR 38 \#3214.

26. - J. Levitt, and M. Shimshoni, Characterization of the set of values $f(n)=[n \alpha], n=$ $1,2, \ldots$, Discrete Math. 2 (1972), 335-345. MR 46 \#1743.

27. - Complementing and exactly covering sequences, J. Comb. Theory Ser. A14 (1973), 8-20. MR 46\#8875.

28. - A characterization of exactly covering congruences, Discrete Math. 4 (1973), 359-366. MR 47 \#4906.

29. - and I. Borosh, A generalization of Wythoff's game, J. Comb. Theory 15 (1973), $175-191$.

30. A. Fraenkel, Further characterizations and properties of exactly covering congruences, Discrete Math. 12 (1975), 93-100.

31. - Complementary systems of integers, in preparation.

32. E. N. Gilbert, Functions which represent all integers, Amer. Math. Monthly 70 (1963), 736-738; Acknowledgement, 1082.

33. H. W. Gould, Problem H-1, Fib. Quart. 1 (1963), 46.

34. - Generalization of a bracket formula of L. Moser, Canad. Math. Bull. 6 (1963), 275-277; Editor's comment, 277-278.

35. - Non-Fibonacci numbers, Fib. Quart. 3 (1965), 177-183. MR 35 \#118.

36. R. L. Graham, On a theorem of Uspensky, Amer. Math. Monthly 70 (1963), 407-409. MR 26 \#6062.

37. - and H. O. Pollak, Note on a nonlinear recurrence related to $\sqrt{ } 2$, Math. Mag. 43 (1970), 143-145. MR 42 \#180.

38. - Covering the positive integers by disjoint sets of the form $\{[n \alpha+\beta]: n=1,2, \ldots\}, \mathrm{J}$. Comb. Theory Ser. A15 (1973), 354-358.

39. H. D. Grossman, A set containing all integers, Amer. Math. Monthly 69 (1962), 532-533.

40. H. Hijikata, On a game of Wythoff (Japanese), Sûgaku 11 (1959/60), 220-222. MR 25 \#42.

41. J. C. Holladay, Cartesian products of termination games, Contributions to the theory of games, Vol. 3, Princeton University Press, New Jersey, 1957. MR 20 \#2236.

42. - Some convergent recursive sequences, homeomorphic identities, and inductively defined complementary sequences, Fib. Quart. 4 (1966), 1-36. MR 36 \#5063.

43. - Some generalizations of Wythoff's game and other related games, Math. Mag. 41 (1968), 7-13.

44. R. Honsberger, Ingenuity in Mathematics, Random House, New York, 1970 (pp. 93-110).

45. L. Kuipers and H. Niederreiter, Uniform Distribution of Sequences, John Wiley and Sons, New York, 1974 (pp. 305-318).

46. J. Lambek and L. Moser, Inverse and complementary sequences of natural numbers, Amer. Math. Monthly 61 (1954), 454-458. MR 16 (1955), p. 17. 
47. W. J. Leveque, editor, Reviews in Number Theory vol. 1, American Mathematical Society, Providence, Rhode Island, 1974 (pp. 331-333). MR \#2040.

48. A. A. Markoff, Sur une question de Jean Bernoulli, Math. Ann. 19 (1882), 27-36.

49. —_ Collected Works (Russian), Izdat. Akad. Nauk. SSSR, Leningrad, 1951 (pp. 13, 15-16).

50. J. C. P. Miller, The sum of the integral parts in an arithmetical progression, Math. Gazette 36 (1952), 234-243. MR 14 (1953), p. 536.

51. D. J. Newman, Problem 5252, Amer. Math. Monthly 71 (1964), 1138. Solutions, ibid., 72 (1965), 1144-1145.

52. I. Niven, Diophantine Approximations, Interscience Tracts in Pure and Applied Mathematics \#14, Interscience Publishers, John Wiley and Sons, New York, c. 1963 (pp. 34-45). MR 26 \#5120.

53. - and H. Zuckerman, An Introduction to the Theory of Numbers, John Wiley and Sons, New York, 2nd ed., 1966. MR 22\#5605.

54. T. H. O'Beirne, Puzzles and Paradoxes, Oxford University Press, London, 1965 (pp. 130-138).

55. Th. Skolem, On certain distributions of integers in pairs with given differences, Math. Scand. 5 (1957), 57-68. MR 19 (1958), p. 1159.

56. - Über einige Eigenschaften der Zahlenmengen $[\alpha n+\beta]$ bei irrationalem $\alpha$ mit einleitenden Bererkungen über einige kombinatorische Probleme, Norske Vid. Selsk. Forh. (Trondheim) 30 (1957), 118-125. MR 19 (1958), pp. 1159-1160.

57. _ Some remarks on the triple systems of Steiner, Math. Scand. 6 (1958), 273-280. MR 19 (1958), p. 1159.

58. N. J. A. Sloane, A Handbook of Integer Sequences, Academic Press, New York, 1973 (pp. 29-30)

59. B. M. Stewart, Theory of Numbers, 2nd ed., MacMillan, New York, 1964 (pp. 76-77). MR 37 \#6232.

ó0. R. Sprague, Ein Satz über Teilfolgen der Reihe der natürlichen Zahlen, Math. Ann. 115 (1938), 153-156.

61. J. V. Uspensky, On a problem arising out of the theory of a certain game, Amer. Math. Monthly 34 (1927), 516-521.

62. — and M. A. Heaslet, Elementary Number Theory, McGraw-Hill, New York, 1939 (p. 98). MR 1 (1940), p. 38.

63. — - On a problem of John Bernoulli. I, II, III, Revista Union Mat. Argentina 11 (1946), 141-154; 164-183; 239-255. MR 8 (1947), pp. 5, 6, 443.

64. — On a problem of John Bernoulli, IV, Revista Union Mat. Argentina 12 (1946), 10-19. MR 8 (1947), p. 443.

65. B. A. Venkov, Elementary Number Theory, Translated and edited by Helen Alderson, Wolters-Noordhoff, Groningen, 1970 (pp. 65-68). MR 42 \#178.

66. I. M. Vinogradov, Elements of Number Theory, translated from the fifth revised edition by S. Kravetz, Dover, 1954 (p. 29). MR 15 (1954), p. 933.

67. W. A. Wythoff, A modification of the game of Nim, Nieuw. Archief voor Viskunde (2), 7 (1907), 199-202.

68. A. M. Yaglom and I. M. Yaglom, Challenging Mathematical Problems with Elementary Solutions, translated by J. McCawley, Jr., revised and edited by B. Gordon, vol. 2, Holden-Day, San Francisco, 1967 (pp. 20, 105-112). MR 29 \#2193.

\section{Department of MATHEMATics \\ UNIVERSITY OF COLORADO \\ BOUlder, COLORADO 80302}

PRESENT AdDRESS:

Department of MATHEMATics

UNIVERSITY OF ILLINOIS

URBANA, ILLINOIS 61801 